\title{
Usefulness of the Computed Tomography Venography for Evaluation of Leg Edema Including Deep Vein Thrombosis in Rehabilitation Patients
}

\author{
Ji Hea Chang, MD ${ }^{1}$, Ho Jun Lee, MD, PhD ${ }^{1}$, Jae Hyun Kwon, $\mathrm{MD}^{2}$, Gi Hyeong Ryu, $\mathrm{MD}^{1}$, \\ Heebong Moon, $\mathrm{MD}^{1}$, Changjae Kim, $\mathrm{MD}^{1}$, Ki Yeon Nam, $\mathrm{MD}^{1}$, Bum Sun Kwon, MD, PhD
}

Departments of ${ }^{1}$ Physical Medicine \& Rehabilitation, ${ }^{2}$ Radiology, Dongguk University College of Medicine, Goyang, Korea

Objective To investigate the usefulness of computed tomography venography (CTV) for evaluation of leg swelling, especially deep vein thrombosis (DVT), in rehabilitation patients.

Methods A hundred twenty-three patients, who had performed CTV performed because of suspected DVT in our clinic, were enrolled. We performed chart reviews retrospectively and categorized CTV findings as follows: DVT distal to inguinal ligament and no compression lesion; DVT proximal to inguinal ligament and no compression lesion; DVT distal to inguinal ligament and anatomical variant (for example, May-Thurner syndrome); DVT due to compression of mass (cancer or cyst); DVT and other incidental abnormal finding; and no DVT and other possible causes of leg swelling.

Results DVTs were found in 65 (53\%) patients. DVTs were found at distal level (thigh or lower leg) to inguinal ligament in 47 patients. DVTs were found at proximal to inguinal ligament, usually undetectable with duplex ultrasonography, in 6 patients. DVTs caused by external compression, such as femoral vein and cancer mass, were found in 12 patients (10\%), which are also not easily detected with duplex ultrasonography. Other various causes of leg edema without DVT were found in $22(18 \%)$ patients.

Conclusion CTV can evaluate more extensively venous problems in the pelvis and abdomen and detect other possible causes of leg swelling. Therefore, CTV can be a useful tool not only for easy detection of DVT but also for evaluating differential diagnosis of leg edema in rehabilitation patients.

Keywords Computed tomography, Edema, Venography, Venous thrombosis

@ This is an open-access article distributed under the terms of the Creative Commons Attribution Non-Commercial License (http://creativecommons.org/ licenses/by-nc/3.0) which permits unrestricted noncommercial use, distribution, and reproduction in any medium, provided the original work is properly cited. Copyright $\odot 2014$ by Korean Academy of Rehabilitation Medicine 


\section{INTRODUCTION}

Leg edema is a common problem. Edema is defined as a palpable swelling caused by an increase in interstitial fluid volume. There are many causes of leg edema, such as deep vein thrombosis, venous insufficiency, pulmonary hypertension, heart failure, and idiopathic edema. In the United States, the most common cause of acute unilateral leg edema is deep vein thrombosis [1].

Deep venous thrombosis (DVT) is part of the spectrum of venous thromboembolism (VTE), with thrombi in the limbs predisposing to pulmonary embolism (PE). The mortality rate for PE following DVT ranges from 5\% to $37 \%$ in untreated patients and is about $6 \%$ among patients treated with anticoagulation after diagnosis [2-5]. PE in stroke patients may cause a higher mortality than that in other clinical settings [6]. DVT is also present in a significant proportion of patients during the rehabilitation phase of stroke and the risk is reported to be greater in those who are more immobile. In a study of $150 \mathrm{pa}$ tients with subacute stage of stroke, bilateral venography revealed DVT in 33\% [7]. The prevalence of DVT in Korea was reported to be $4.17 \%$ in rehabilitation patients with hemiplegia [8] and 5.7\% in spinal cord injury patients [9]. Therefore, detection and early treatment of DVT are very important for patients in rehabilitation units. Despite accounting for 300,000 to 600,000 hospitalizations in the United States annually, venous thromboembolic disease is quite difficult to diagnose clinically [10]. Due to the deficit of sensory function and communication difficulty of patients in rehabilitation units, leg swelling is sometimes found late and the clinical diagnosis of DVT can be difficult.

Conventional venography, widely accepted as the best test for detection of lower extremities DVT, is invasive and is, recently, less routinely used. Duplex ultrasonography, a non-invasive test without radiation and contrast material, has replaced conventional venography in the investigation of patients with suspected DVT [11], but duplex ultrasonography of the calf veins was time-consuming and dependent on the operator's experience. In addition, the small isolated thrombi confined within the calf vein were easily ignored [12].

Due to the development of technology the venography using multidetector computed tomography with enhancement was introduced. Reported sensitivity of com- puted tomography venogram (CTV) has varied between $89 \%$ and $100 \%$, whereas specificity has ranged from $94 \%$ to $100 \%$ [11]. Goodman et al. [13] reported $95.5 \%$ concordance between CTV and venous compression sonography for the diagnosis or exclusion of DVT and similar diagnostic results of CTV and sonography. CTV and sonography were similar; CTV was reported to be an accurate modality for the assessment of femoropopliteal DVT in comparison with lower extremity duplex ultrasonography and CTV was reported to have the advantage of providing information of proximal vasculature, including the inguinal ligaments and inferior vena cava [14]. We supposed that CTV can provide more information about proximal veins in abdomen and pelvis and various conditions of lower leg vasculature than duplex ultrasonography.

This study is purposed to investigate the usefulness of CTV for evaluation of leg edema that was suspected to be DVT.

\section{MATERIALS AND METHODS}

We retrospectively reviewed the medical records of patients who had CTV with the impression of DVT because of acute leg edema from 2009 to 2012. Data of 123 patients were collected. We included the patients who underwent CTV for the initial evaluation just after leg swelling. We excluded six patients who did not have D-dimer test. We investigated the demographic and clinical characteristics (age, gender, body mass index [BMI], primary diagnosis, and underlying diseases). In addition, we obtained clinical data about characteristics of DVT, such as level of DVT presence of PE, location, and anatomic level of DVTproximal/distal to inguinal ligament-and risk factors including surgery (recent), trauma (major trauma or lowerextremity injury), cancer, immobility, lower-extremity paresis, venous compression (tumor, hematoma, arterial abnormality), age, obesity, central venous catheterization, previous VTE, and antiphospholipid syndrome [15]. The subjects were 50 men and 67 women, and their mean ages were $63.0 \pm 16.4$ years. Underlying diseases before performing CTV were various-stroke, cancer, spinal cord injury, venous problem including varicose vein, lymphedema, arterial thrombosis and just lower extremity swelling.

CTV was performed on a 64-channel multidetector row 
helical CT scanner (SOMATOM Sensation 64; Siemens, Forchheim, Germany) with about $140 \mathrm{~mL}$ of contrast medium (Ultravist; Bayer Schering Pharma AG, Berlin, Germany) at a rate of $4 \mathrm{~mL} / \mathrm{sec}$ by means of a power injector through a 18- to 20-guage intravenous catheter in the antecubital vein. CTV was started three minutes after contrast medium injection from foot to diaphragm with the following parameters: collimation of $64 \times 0.6 \mathrm{~mm}$ in fast mode, rotation time 0.37 seconds per gantry rotation, and a pitch of 0.7. Five-millimeter-thick axial images were reconstructed without intersection gap. DVT was defined as a low-attenuating partial or complete intraluminal filling defect surrounded by a high-attenuating ring of enhanced blood that was seen on at least two consecutive transverse images [16].

D-dimer is a degradation product of cross-linked fibrin blood clot. Elevated levels of D-dimer occurred with acute thrombosis. The D-dimer test is used as a screening test for VTE, but this test does not discriminate between physiologic or pathologic thrombi [17]. The D-dimer test was conducted at the same time with CTV. For the D-dimer test, a venous blood sample was collected into a test tube containing sodium citrate, and tested with the D-Dimer PLUS (Siemens). A D-dimer level above $324 \mu \mathrm{g} /$ L was considered positive.

We categorized CTV findings as follows: 1) DVT distal to inguinal ligament and no compression lesion, 2) DVT proximal to inguinal ligament (inferior vena cava and renal vein), 3) DVT due to anatomical variant (for example,
May-Thurner syndrome), 4) DVT due to compression of mass (cancer or cyst), 5) DVT and incidental abnormal finding of other organ, and 6) no DVT with other possible causes of leg swelling.

Statistical analyses were performed using t-test for continuous numeric variables and chi-square test for not continuous variables in order to compare the differences between the DVT group and no DVT group. We performed ANOVA test to identify the difference of D-dimer values among DVT distal to inguinal ligament group, DVT proximal to inguinal ligament group, no DVT with other cause group and no DVT without other cause group. All statistical analyses were performed using SPSS-K ver 20.0 (IBM SPSS Inc., Armonk, NY, USA).

\section{RESULTS}

Among all 117 patients who underwent CTV, DVT was found in 65 patients. As described in methods, we classified patients with DVT into five groups. Group 1, DVT distal to inguinal ligament and no compression lesion, had 47 patients, and most patients were included in group 1. Group 2, DVT proximal to inguinal ligament and no compression lesion, had six patients. Group 3, DVT was caused by venous compression with anatomical variant, had eight patients. Group 4, DVT with cancer mass, had three patients. The location of DVTs in groups 3 and 4 was similar to group 2 in that they were placed proximal to inguinal ligament. Therefore, CTV could detect, not
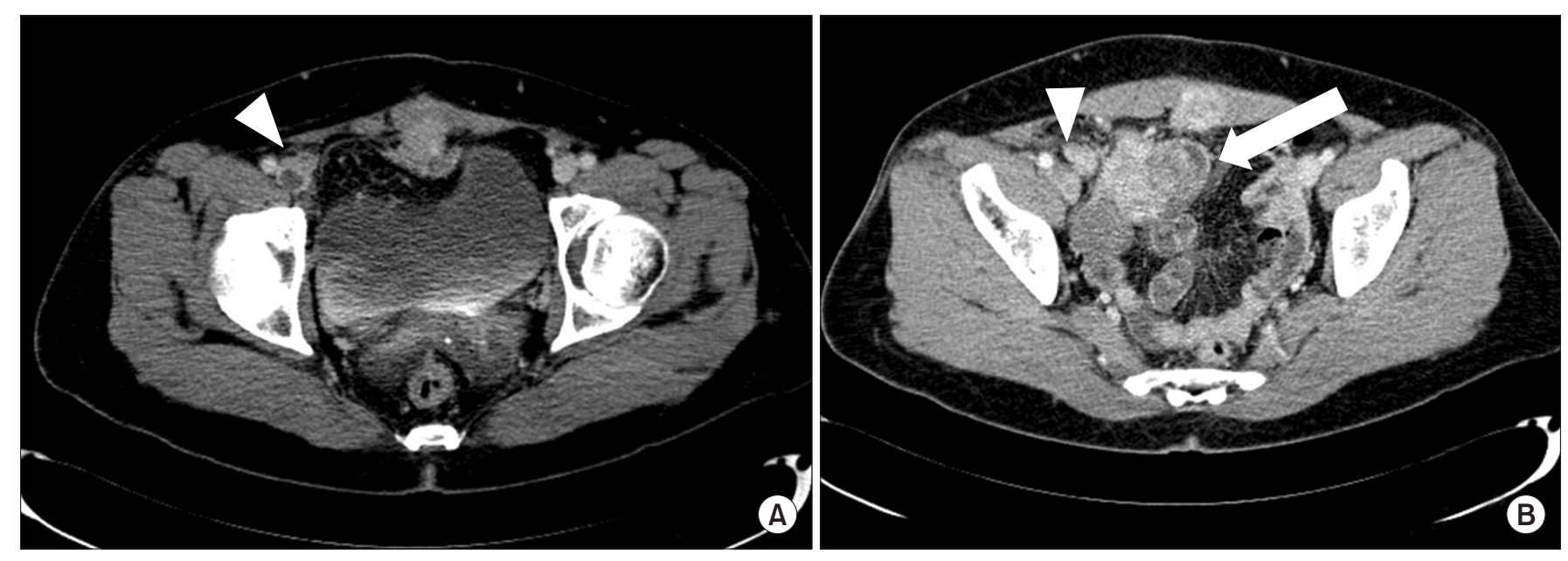

Fig. 1. The computed tomography venography reveals low signal intensity lesion, which means deep vein thrombosis (DVT), inside of right iliac vein (arrowhead) (A) and the well enhancing mass lesions at right lower abdomen (white arrow) compress right external iliac vein (arrowhead) proximal to DVT lesion (B). 
Table 1. Classification of CTV findings in patients with DVT

\begin{tabular}{clc}
\hline Group & \multicolumn{1}{c}{ CTV findings } & D-dimer $(\boldsymbol{\mu g} / \mathbf{L})$ \\
\hline 1 & DVT distal to inguinal ligament and no compression lesion $(\mathrm{n}=47,38 \%)$ & $1,083.9 \pm 1,347.0$ \\
\hline 2 & DVT proximal to inguinal ligament and no compression lesion $(\mathrm{n}=6,5 \%)$ & $718 \pm 603.4$ \\
\hline 3 & DVT distal to inguinal ligament and anatomical variant & \\
& May-Thurner syndrome $(\mathrm{n}=7,6 \%)$ & $3,577.7 \pm 2,200.7$ \\
\hline & Right common femoral vein stenosis $(\mathrm{n}=1,1 \%)$ & 250 \\
\hline & Compression by cancer (n=3, 3\%) & $2,917.5 \pm 3,991.6$ \\
& Cancer recurrence (n=2, 2\%) & 1,995 \\
\hline & Growth of existing cancer mass (n=1, 1\%) & 1,028 \\
\hline
\end{tabular}

Values are presented as mean \pm standard deviation.

CTV, computed tomography venography; DVT, deep vein thrombosis.
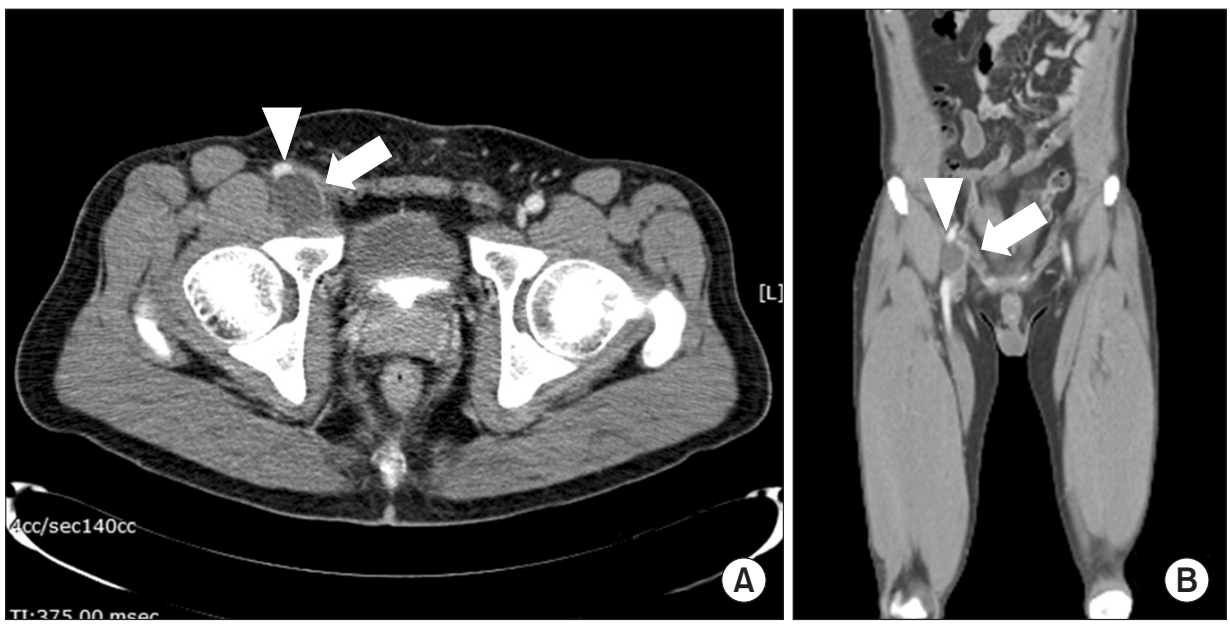

Fig. 2. This image shows low density $2.8 \times 2.9 \times 2.1 \mathrm{~cm}$ sized mass lesion (white arrow) in posterolateral area to right common femoral vein (arrowhead) in axial view (A) and the mass (white arrow) compresses right common femoral vein (arrowhead) in coronal view (B). only DVT itself, but also underlying lesions causing DVT in 11 patients. In one patient, CTV revealed the DVT at right iliac vein and lower abdomen mass, which was later diagnosed as recurrence of uterus cancer, compressing right external iliac vein (Fig. 1). Group 5 included one patient, and cholangiocarcinoma was incidentally found in addiction to the location of his DVT so that the patient was referred to the department of general surgery (Table 1).

DVT was not found in $52(47 \%)$ patients, and other various causes of leg edema without DVT were found in 22 (18\%) patients (group 6). Thigh and gluteal muscle problems were found in seven patients; thigh muscle tear with hematoma were found in five patients. Muscle abscess was found in one patient and gluteal muscle hematoma after hip surgery was found in one patient. Superficial vein dilatations were found in seven patients and might be related to varicose vein or venous insuffi- ciency. Venous compressions in pelvis were found in two patients: ganglion cyst compressing femoral vein (Fig. 2) and anatomical variant without DVT. CTV also detected incidental abnormal findings not related to DVT and leg edema in six patients-leg multiple artery stenosis (two patients), abdominal aorta aneurysm with multiple leg artery stenosis (one patient), right iliac artery focal aneurysm (one patient), kidney lesion (acute pyelonephritis, one patient) and calf muscle mass (one patient).

The clinical characteristics and risk factors were compared between DVT group and no DVT group. DVT group were female predominant $(n=40)$ with a mean age of 63 years. DVT was not found in 22 patients with a mean age of 54 years and both male and female had similar numbers. BMI was $23.8 \pm 4.36 \mathrm{~kg} / \mathrm{m}^{2}$ in DVT group and $25.0 \pm 4.12 \mathrm{~kg} / \mathrm{m}^{2}$ in no DVT group. Between DVT group and no DVT group there were no statistically significant 
Table 2. Comparisons of clinical characteristics and risk factors in patients with DVT and without DVT

\begin{tabular}{lccc}
\hline & $\begin{array}{c}\text { DVT (+) } \\
(\mathbf{n = 6 5 )}\end{array}$ & $\begin{array}{c}\text { DVT (-) } \\
(\mathbf{n = 5 2 )}\end{array}$ & p-value \\
\hline Gender (male:famale) & $25: 40$ & $25: 27$ & 0.23 \\
Age (yr) & $63.0 \pm 17.4$ & $64.0 \pm 14.8$ & 0.95 \\
BMI $\left(\mathrm{kg} / \mathrm{m}^{2}\right)$ & $23.8 \pm 4.36$ & $25.0 \pm 4.12$ & 0.15 \\
LE paralysis & $22(35.0)$ & $21(40.0)$ & 0.21 \\
Immobility & $14(22.0)$ & $16(31.0)$ & 0.18 \\
Surgery & $4(16.0)$ & $3(6.0)$ & 0.06 \\
Trauma & $3(6.0)$ & $0(0)$ & $0.04^{*}$ \\
Cancer & $10(5.0)$ & $4(8.0)$ & $<0.01^{*}$ \\
Previous VTE & $1(2.0)$ & $0(0)$ & $0.01^{*}$ \\
No. risk factors & $9(14.0)$ & $8(15.0)$ & 0.08 \\
\hline
\end{tabular}

Values are presented as mean \pm standard deviation or number (\%).

DVT, deep vein thrombosis; BMI, body mass index; LE paralysis, lower extremity paralysis including hemiplegia, paraplegia, and tetraplegia; VTE, venous thromboembolism.

${ }^{*} \mathrm{p}<0.05$, significant differences exists between DVT group and no DVT group.

differences in age, gender, and BMI. Risk factors of DVT in DVT group were similar to those in no DVT group. In DVT group, nine patients had no risk factors of DVT; however, lower extremity paralysis, one of the most usual risk factors of DVT in rehabilitation patients, were $40 \%$ in no DVT group. Cancer and trauma were a significantly higher proportion in DVT group than in no DVT group, despite the small numbers (Table 2). We classified patients into four groups: distal DVT group (groups 1 and 5), proximal DVT group (groups 2, 3, and 4), no DVT with other possible cause of leg edema (group 6), and no DVT. The distributions of risk factors in these four groups were also similar, as shown in Table 2 and Fig. 3.

D-dimer was used as screening tool for DVT. The level of D-dimer was significantly higher in the DVT group $(1,377.4 \pm 1,647.37 \mu \mathrm{g} / \mathrm{L})$ than no DVT group (660.5 \pm $1,249.83 \mu \mathrm{g} / \mathrm{L})(\mathrm{p}=0.011)$. The $\mathrm{D}$-dimer values were compared among four groups: distal DVT group, proximal DVT group, no DVT with other possible cause of leg edema, and no DVT. The D-dimer was significantly higher in proximal DVT group (groups 2, 3, and 4) than the other group (Fig. 4).

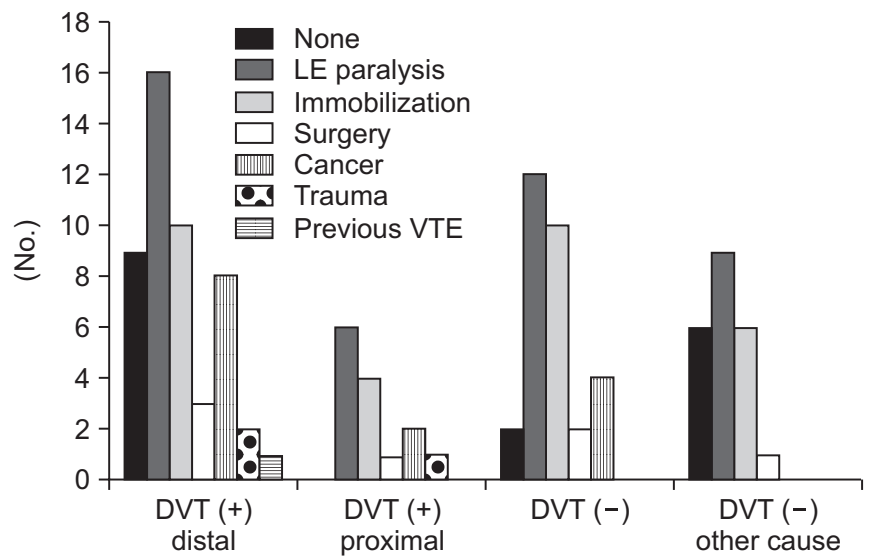

Fig. 3. The graph shows distributions of risk factors of VTE among four groups: DVT (+) distal, DVT distal to inguinal ligament; DVT (+) proximal, DVT proximal to inguinal ligament (in abdomen/pelvis) or with compression of other structures; DVT (-), no DVT detected; and DVT (-) other causes, no DVT and other causes of leg swelling detected). The relative proportion of LE paralysis in DVT (-) and DVT (-) with other cause groups was higher, similar to DVT (+) group. Therefore in the patients with LE paralysis usually present in the department of rehabilitation there are various causes of leg swelling and some causes can be found more easily by computed tomography venography than other study. DTV, deep vein thrombosis; VTE, venous thromboembolism; LE, lower extremity.

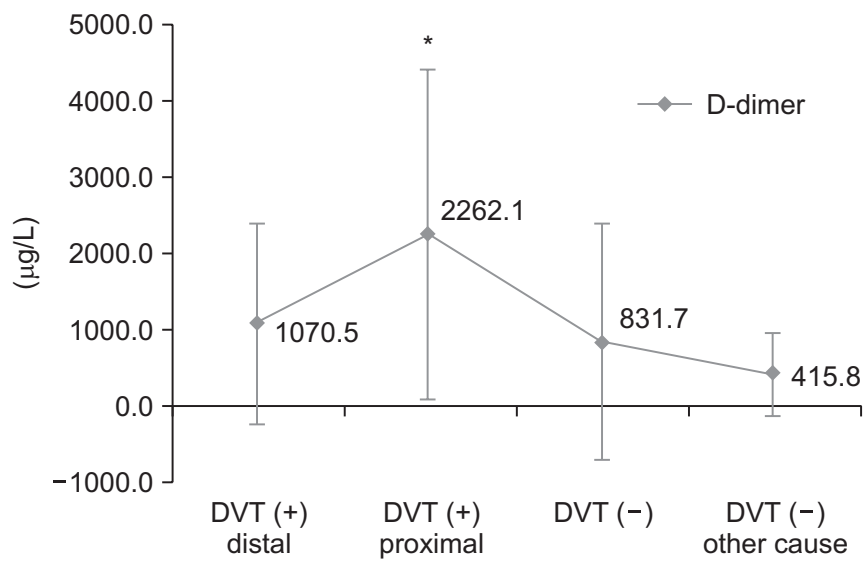

Fig. 4. This graph shows the D-dimer values of four groups. D-dimer values of deep vein thrombosis (DVT) proximal group (groups 2, 3, and 4) were significantly different from those of the other three groups. ${ }^{*} p<0.05$, result from ANOVA with post-hoc analysis. 


\section{DISCUSSION}

The data of this study showed the various usefulness of CTV in leg edema with suspect of DVT. The first advantage is the detection of proximal DVT (proximal to inguinal ligament), which cases were 16 subjects and occupied $33 \%$ of all cases of DVT and of which 11 patients were also diagnosed to have PE. The location of DVTs in group 2 was inferior vena cava and common iliac veins, and DVT of these sites is supposed to be hard to detect with duplex ultrasonography. As previously mentioned, the duplex ultrasonography has been considered as a valuable screening tool for detection of DVT, and CTV and duplex ultrasonography have similar specificity and sensitivity [11]. We think that the limit of duplex ultrasonography is the difficulty in examining veins in abdomen and pelvis. In aged persons, great vessels in the abdomen and pelvis are usually tortuous and abdomen distension is frequent due to decreased skin elasticity. Moreover, in obese or edematous persons, abundant subcutaneous fatty tissue usually disturbs the examination of deep structures, including vessels because of higher echogenicity.

The second advantage of CTV is the ability of detecting direct cause of DVT. In group 3 and group 4, CTV showed the direct cause of DVT, including anatomical variant and compressing mass. In group 3, the cause of DVT in seven patients was the anatomical variant, which is called May-Thurner syndrome. May-Thurner syndrome is a condition in which the left iliac vein is compressed by the right iliac artery. It was hypothesized to be due to chronic compression of the left iliac vein between the right common iliac artery and L5 vertebral body. Direct visualization of the iliac veins by duplex ultrasound in the pelvis can be difficult because of overlying pelvic organs and bowel gas [18]. Chung et al. [19] reported that 45 of 56 patients had a detectable abnormality that was at least partly responsible or acute DVT with spiral CTV, and they also reported that 27 of 44 patients with acute iliofemoral DVT had findings of May-Thurner syndrome detectable on CT. In group 4, cancer mass compressed the vein and that was the direct cause of DVT. In two patients, who had been diagnosed in the remission state from underlying cancer, the mass compressing vein in pelvis was detected, which were later identified as cancer recurrence. One patient who had a uterus cancer was referred to our department for leg swelling with suspect of lymphedema, but relatively sudden swelling brought the need for differential diagnosis of DVT and the CTV revealed the existing cancer mass extension causing compression of iliac vein.

The third advantage is the incident detection of other pathologic finding in the abdomen. In our analysis, there was just one case detecting cholangiocarcinoma. At that time the patient had no symptoms and signs in abdomen as well as no jaundice. Cancer is one of major risk factors of DVT [15], and this patient had a hidden risk factor.

The fourth advantage is the detection of other possible causes of leg edema besides DVT. Group 6 had 22

Table 3. No DVT and other causes of leg edema found in CTV without DVT

\begin{tabular}{lc}
\hline \multicolumn{1}{c}{ CTV findings } & No. of patients (\%) \\
\hline $\begin{array}{l}\text { Thigh and gluteal muscle problems (muscle tears with hematoma, postoperative hematoma } \\
\text { after hip surgery) }\end{array}$ & $7(6.0)$ \\
\hline Superficial vein dilatations (might be related to varicose vein or venous insufficiency) & $7(6.0)$ \\
\hline Vein compressions in pelvis & $1(1.0)$ \\
\hline Ganglion cyst compressing femoral vein & $1(1.0)$ \\
\hline Anatomical variant without DVT & $2(2.0)$ \\
\hline Incidental abnormal findings not related to DVT and leg swelling & $1(1.0)$ \\
\hline Leg multiple artery stenosis & $1(1.0)$ \\
\hline Abdominal aorta aneurysm with multiple leg artery stenosis & $1(1.0)$ \\
\hline Right iliac artery focal aneurysm & $1(1.0)$ \\
\hline Right kidney lesion & \\
\hline Calf muscle mass &
\end{tabular}

DVT, deep vein thrombosis; CTV, computed tomography venography. 
patients and occupied $18 \%$ of all subjects and $42 \%$ of all no DVT group. Especially seven patients (32\% in group 6) had a muscle problem in thigh or buttock. This point is very important to patients with paraplegia or tetraplegia. Causes of leg swelling are various and differential diagnosis of leg swelling in spinal cord injury patients are DVT, muscle tear, hematoma, heterotopic ossification and fracture [20]. Among them, DVT is most fatal, and the detection of the presence or absence of DVT is the first step with duplex ultrasonography. However, with duplex ultrasonography, there is certain limitation in detecting all other differential diagnosis. CTV can show the abnormality of bone, muscle, and other abnormality of various structures, including muscle abscess, ganglion cyst, hematoma from muscle tear, and heterotopic ossification (Table 3). The patients with complete paraplegia or tetraplegia often cannot complain of pain in the lower extremity from muscle tear, which often occurs during stretching exercise, during transfer, and at falling. They or their caregivers often find the swelling of leg and then visit the hospital. Therefore, the patients of lower extremity paralysis need an expansive examination of lower extremity and pelvis to find the cause of leg edema, and the CTV is the most suitable examination tool for them. One case in group 6 was ganglion cyst of hip joint compressing right femoral vein. The patient came to the hospital with complaint of just right leg swelling without other symptoms and any underlying disease. CTV found a low signal intensity mass compressing right femoral vein (Fig. 2), and surgeons removed the mass, which was concluded as ganglion cyst rising from hip joint, and simultaneously performed balloon angioplasty. At three months after surgery and intervention, the swelling of leg decreased to the similar size of contralateral side. Therefore, CTV can find other various causes of leg edema besides DVT.

D-dimer is a specific fibrinolysin-mediated breakdown product of cross-linked fibrin and is nowadays usually used as a screening tool for DVT. Although this assay has a high sensitivity, it has low specificity. It is also elevated in a number of other common clinical situations, such as liver disease, pregnancy, recent trauma or surgery, cancer, massive bleeding, multiple traumatic injuries, and so on. D-dimer concentration increases with age and its specificity for embolism decreases [21]. In rehabilitation inpatients, D-dimer testing can be used as a routine screening test or to assess cases of suspected DVT [22].
Several studies indicate that the D-dimer assay can be applied as a simple, inexpensive screening test for VTE. A cutoff value of D-dimer in our hospital was $340(\mu \mathrm{g} /$ L). Harvey et al. [23] showed that a D-dimer level $\leq 1,092$ $\mathrm{ng} / \mathrm{mL}$ can exclude the presence of DVT in stroke rehabilitation patients. The D-dimer cutoff point up to 1,092 $\mathrm{ng} / \mathrm{mL}$ showed both sensitivity and negative predictive value to $100 \%$ but specificity to $66 \%$ and positive predictive value to $31 \%$. D-dimer values of each patient were various and the analysis with comparison DVT group is statistically higher than no DVT group, which is similar to previous reports. Among the four groups, D-dimer level in proximal DVT group is statistically higher than in the other groups. Therefore, with consideration of low specificity [22], when D-dimer value is high, we should suspect DVT located in both iliofemoral vein and proximal veins, and sometimes, we should suspect DVT associated with cancer.

Despite the absence of direct comparison with duplex ultrasonography, we suggest that CTV has a good advantage of detection of proximal DVT and also the abnormality of other structure or mass compressing the vein located in the abdomen and pelvis, and CTV is thought to provide more information of abdomen and pelvic lesion, which is a possible cause of acute leg edema, than duplex ultrasonography.

This study has several limitations. The first limitation is that this study was a retrospective review and we did not compare the diagnostic capability of CTV with duplex ultrasonography. Therefore, we cannot definitely insist that CTV is more useful in diagnosis of DVT than duplex ultrasonography. If we performed CTV and duplex ultrasonography in the same patient at similar times the diagnostic advantages of CTV over duplex ultrasonography, which are described above, could have been proven. The second limitation is the heterogeneity and small numbers of subjects. The subjects were included with just leg swelling and their underlying disease were various from healthy persons to tetraplegia patients. If we had included more subjects, we could have analyzed various characteristics of DVT occurrence and the influence of risk factors in each group of patients according to underlying disease and the severity of paralysis. For example, previous studies about characteristics of DVT in stroke or spinal cord injury patients used either venography or duplex ultrasonography as a diagnostic tool. However, if we 
performed CTV as a diagnostic tool in many patients with stroke or spinal cord injury, the prevalence or incidence of DVT could be reported higher than for previous studies in Korea.

In conclusion, CTV can evaluate venous problems in pelvis and abdomen more extensively and can be performed more easily and for shorter duration than duplex ultrasonography. Moreover, CTV can detect other possible causes of leg swelling, which are difficult to obtain with ultrasonography. Therefore, CTV is a very useful diagnostic tool for leg swelling, especially in rehabilitation patient with stroke and spinal cord injury, who usually suffer from swelling in paretic leg or asymptomatic DVT because it is hard for such patients to complain of leg swelling.

\section{CONFLICT OF INTEREST}

No potential conflict of interest relevant to this article was reported.

\section{REFERENCES}

1. Ely JW, Osheroff JA, Chambliss ML, Ebell MH. Approach to leg edema of unclear etiology. J Am Board Fam Med 2006;19:148-60.

2. Warlow C, Ogston D, Douglas AS. Deep venous thrombosis of the legs after stroke. Part 2: Natural history. Br Med J 1976;1:1181-3.

3. Kelly J, Rudd A, Lewis R, Hunt BJ. Venous thromboembolism after acute stroke. Stroke 2001;32:262-7.

4. Byrne JJ. Phlebitis; a study of 748 cases at the Boston City Hospital. N Engl J Med 1955;253:579-86.

5. Collins R, Scrimgeour A, Yusuf S, Peto R. Reduction in fatal pulmonary embolism and venous thrombosis by perioperative administration of subcutaneous heparin: overview of results of randomized trials in general, orthopedic, and urologic surgery. N Engl J Med 1988;318:1162-73.

6. Wijdicks EF, Scott JP. Pulmonary embolism associated with acute stroke. Mayo Clin Proc 1997;72:297-300.

7. Bromfield EB, Reding MJ. Relative risk of deep venous thrombosis or pulmonary embolism post-stroke based on ambulatory status. Neurorehabil Neural Repair 1988;2:51-7.

8. Ko HY, Shin YB, Jho SK. Incidence of deep vein throm- bosis in spinal cord injury. J Korean Acad Rehabil Med 2005;29:359-64.

9. Han TR, Lim SJ, Lee HJ. Deep vein thrombosis in rehabilitation inpatients. J Korean Acad Rehabil Med 2001;25:827-35.

10. Anderson FA Jr, Wheeler HB, Goldberg RJ, Hosmer DW, Patwardhan NA, Jovanovic B, et al. A populationbased perspective of the hospital incidence and casefatality rates of deep vein thrombosis and pulmonary embolism: the Worcester DVT Study. Arch Intern Med 1991;151:933-8.

11. Lim KE, Hsu WC, Hsu YY, Chu PH, Ng CJ. Deep venous thrombosis: comparison of indirect multidetector CT venography and sonography of lower extremities in 26 patients. Clin Imaging 2004;28:439-44.

12. Byun SS, Kim JH, Kim YJ, Jeon YS, Park CH, Kim WH. Evaluation of deep vein thrombosis with multidetector row $\mathrm{CT}$ after orthopedic arthroplasty: a prospective study for comparison with Doppler sonography. Korean J Radiol 2008;9:59-66.

13. Goodman LR, Stein PD, Matta F, Sostman HD, Wakefield TW, Woodard PK, et al. CT venography and compression sonography are diagnostically equivalent: data from PIOPED II. AJR Am J Roentgenol 2007;189:1071-6.

14. Loud PA, Katz DS, Klippenstein DL, Shah RD, Grossman ZD. Combined CT venography and pulmonary angiography in suspected thromboembolic disease: diagnostic accuracy for deep venous evaluation. AJR Am J Roentgenol 2000;174:61-5.

15. Geerts WH, Bergqvist D, Pineo GF, Heit JA, Samama $\mathrm{CM}$, Lassen MR, et al. Prevention of venous thromboembolism: American College of Chest Physicians Evidence-Based Clinical Practice Guidelines (8th Edition). Chest 2008;133(6 Suppl):381S-453S.

16. Cham MD, Yankelevitz DF, Shaham D, Shah AA, Sherman L, Lewis A, et al. Deep venous thrombosis: detection by using indirect CT venography. The Pulmonary Angiography-Indirect CT Venography Cooperative Group. Radiology 2000;216:744-51.

17. Knapton S, Pandian G. Lower limb peripheral vascular disease. In: Braddom RL, editor. Physical medicine and rehabilitation. 4 th ed. Philadelphia: Saunders; 2011. p. 1347-70.

18. Ley EJ, Hood DB, Leke MA, Rao RK, Rowe VL, Weaver FA. Endovascular management of iliac vein occlusive 
disease. Ann Vasc Surg 2004;18:228-33.

19. Chung JW, Yoon CJ, Jung SI, Kim HC, Lee W, Kim YI, et al. Acute iliofemoral deep vein thrombosis: evaluation of underlying anatomic abnormalities by spiral CT venography. J Vasc Interv Radiol 2004;15:249-56.

20. Bryce TN, Ragnarsson KT, Stein AB, Biering-Sorensen F. Spinal cord injury. In: Braddom RL, editor. Physical medicine and rehabilitation. 4th ed. Philadelphia: Saunders; 2011. p. 1293-1346.

21. Hou H, Ge Z, Ying P, Dai J, Shi D, Xu Z, et al. Biomark- ers of deep venous thrombosis. J Thromb Thrombolysis 2012;34:335-46.

22. Akman MN, Cetin N, Bayramoglu M, Isiklar I, Kilinc $\mathrm{S}$. Value of the D-dimer test in diagnosing deep vein thrombosis in rehabilitation inpatients. Arch Phys Med Rehabil 2004;85:1091-4.

23. Harvey RL, Roth EJ, Yarnold PR, Durham JR, Green D. Deep vein thrombosis in stroke: the use of plasma D-dimer level as a screening test in the rehabilitation setting. Stroke 1996;27:1516-20. 